\title{
Electron beam excitation of CSRR loaded waveguide for Cherenkov radiation
}

\author{
Emmy Sharples ${ }^{a, b}$ and Rosa Letizia ${ }^{b, a}$ \\ ${ }^{a}$ The Cockcroft Institute, Sci-Tech Daresbury, Warrington, United Kingdom; \\ ${ }^{b}$ Department of Engineering, Lancaster University, Bailrigg, Lancaster, United Kingdom
}

\begin{abstract}
A novel metamaterial structure is presented for applications as a backward propagating Cherenkov source or Cherenkov detector. The structure comprises of a complementary split ring resonator (CSRR) metasurface loaded waveguide, which exhibits left handed behaviour between 5-6 GHz. When the left handed, TM-like mode couples with an incident electron beam, backward propagating Cherenkov radiation is observed. The structure is suitable for beam-based applications, exhibiting strong beam coupling parameters and significant excitation of longitudinal wakefields. Three dimensional particle in cell simulations are performed to identify a suitable beam for operation. High and low energy beams, with different bunch dimensions from the literature, are considered and compared to investigate the nature of the beam-wave interaction this structure can support, and to identify any required modification before beam tests can be performed. This structure can lead to new solutions for non-destructive beam diagnostics, wakefield acceleration and backward wave oscillators which can potentially be scaled to higher frequency ranges.
\end{abstract}

Keywords: Metamaterials, complementary split ring resonators, Cherenkov radiation, loaded waveguide, particle accelerators

\section{INTRODUCTION}

Metamaterial devices and metamaterial loaded waveguides provide a novel alternative to dielectric structures as electron beam driven devices for the generation of coherent radiation. In a left handed medium ${ }^{1}$ such as the complementary split ring resonator (CSRR) loaded waveguide we have recently proposed, ${ }^{2}$ backward propagation of electromagnetic waves is observed. Thanks to this property, when an electron travels faster than photons in this artificial medium, the generation of reverse Cherenkov radiation ${ }^{3}$ can be observed. These structures are very attractive as alternative Cherenkov detectors and sources.

Conventional electron-beam driven coherent sources include synchrotrons, ${ }^{4}$ free-electron lasers (FELs) ${ }^{5}$ backward wave oscillators (BWOs) and dielectric loaded waveguides. Synchrotrons and FELs provide higher power radiation but also require large scale facilities which limits their availability and consequently the impact this technology can bring to everyday applications. Smaller scale radiation sources such as BWOs and dielectric loaded waveguides ${ }^{6}$ have recently regained attention in the search for a satisfactory compromise between high power operation and compactness of the device. Due to the nature of the phenomenon involved, dielectric loaded waveguides are typically used to exploit the Cherenkov effect of coherent radiation generation. Although useful to support high frequency operation, dielectrics can suffer from accumulation of charge, breakdown ${ }^{7}$ at the vacuum/dielectric gaps where the fields are enhanced and thermal management issues. The use of an all metallic metamaterial structure can prevent these practical issues, while providing an effective means for dispersion engineering, to mimic the behaviour of dielectrics. The typical dispersion of a metallic rectangular waveguide can be modified by loading it with a metamaterial. The composite structure can be tailored to support both forward and backward propagating Cherenkov radiation.

In our previous work, we successfully proposed a metamaterial loaded waveguide made of metal suitable for

Further author information: (Send correspondence to E. Sharples)

E-mail: emmy.sharples@cockcroft.ac.uk, 

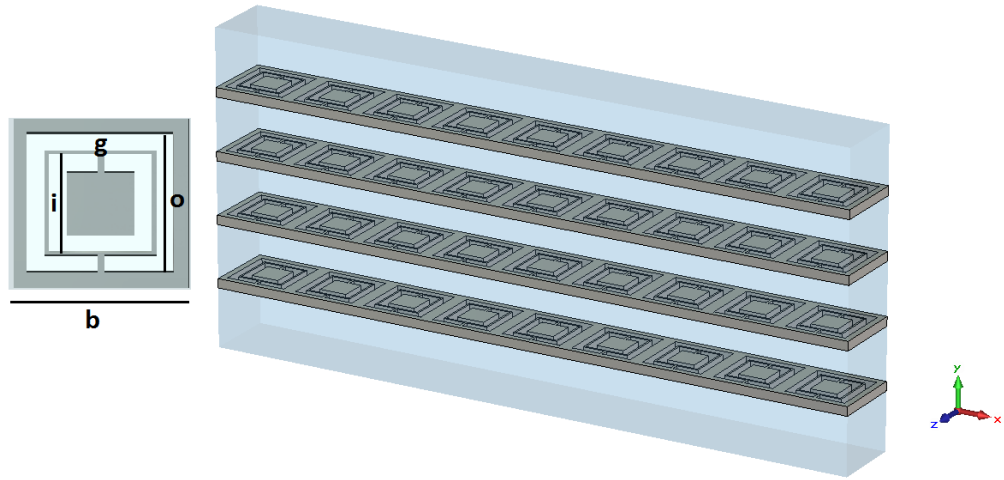

Figure 1. The unit cell and a segment of the loaded waveguide, with geometric dimensions labelled.

accelerator applications ${ }^{2}$ as shown in Figure. 1 and discussed its wakefield response. ${ }^{8}$ To reduce the surface current build up on the metasurface sheets, the thickness was optimised to $1 \mathrm{~mm}$, this increased the fabrication suitability of the structure allowing for larger segments to be machined. The increased sheet thickness had the additional benefit of reducing the number of hybrid modes within the structure allowing for easier coupling to the desired TM-like mode. ${ }^{9}$ For this structure the TM-like mode is the operating mode and a specific coupler has been designed to allow beam and RF loading into the structure to occur for the correct mode.

In this paper full three-dimensional particle in cell (PIC) simulations of the proposed structure are performed to provide a comprehensive understanding of the structure's response to realistic electron beams. PIC simulations allow for the analysis of the electromagnetic waves being excited when an electron beam propagates through the structure. Through the use of field probes and three dimensional field monitors it is possible to identify the frequency of the generated field and the field profile in space, to ensure that the correct mode is being excited. A number of beams ranging in energy from $100 \mathrm{keV}$ to $20 \mathrm{GeV}$ with large $(2 \mathrm{~mm})$ and small $(250 \mu \mathrm{m})$ spot sizes are considered for comparison of the structure performance.

This paper is organized as follows: an overview of the loaded waveguide structure is given in Section 2. The response of the structure when electron beams from VELA, AWAKE, and Kimball, propagate through it are given in Section 3. Finally, conclusions are presented in Section 4.

\section{THE CSRR LOADED WAVEGUIDE}
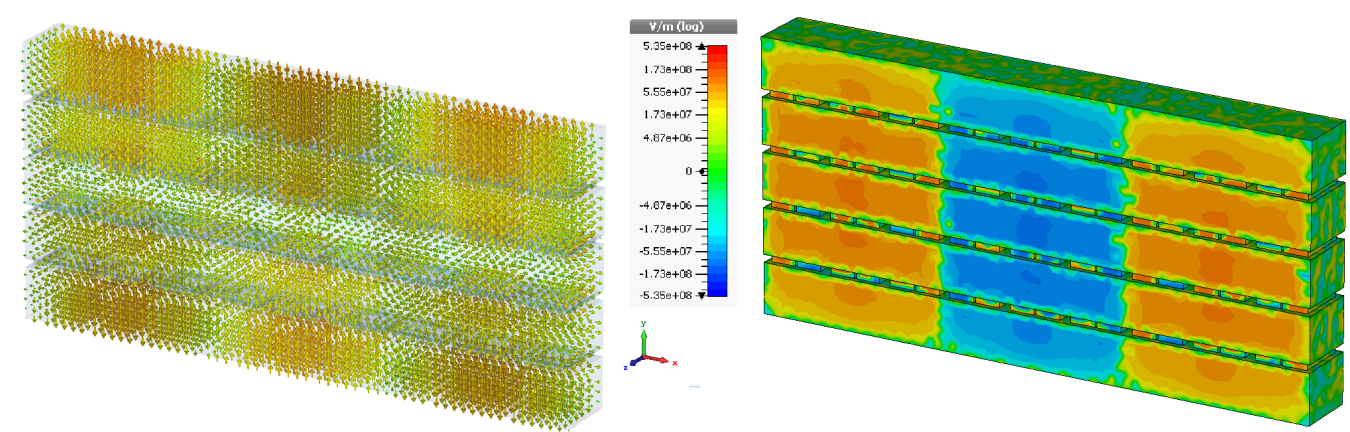

Figure 2. The three dimensional field profile of the TM-like mode and the spatial profile of the $E_{z}$ component of the TM-like mode found at $5.86 \mathrm{GHz}$ for a phase advance of $10^{\circ}$ in the loaded waveguide structure.

Figure. 1 shows the unit cell of the complementary split ring resonator (CSRR) metamaterial used, and a single strip of the loaded waveguide. The loaded waveguide structure comprises of four layers of CSRR metasurface, $1 \mathrm{~mm}$ thick, the geometric dimensions of the CSRR are given in Table. 2. The metasurfaces are loaded into a metallic WR-284 waveguide, $72 \mathrm{~mm}$ by $34 \mathrm{~mm}$, this allows for a separation of $5.81 \mathrm{~mm}$ between 
the four layers, each sheet is 9 resonators wide in the transverse direction and is truncated to 40 resonators in the longitudinal direction. The sheet thickness of $1 \mathrm{~mm}$ is used to reduce the surface current build up in the structure and to improve the fabrication suitability, it is also found to reduce the number of hybrid modes within the structure making coupling to this mode easier. ${ }^{9}$

Table 1. The geometric parameters of the CSRR unit cell

\begin{tabular}{lcr}
\hline Parameter & Symbol & \multicolumn{2}{r}{ Value (mm) } \\
\hline \hline & & 8 \\
Unit Cell & $\mathrm{b}$ & 6.6 \\
Outer Ring & $\mathrm{o}$ & 4.6 \\
Inner Ring & $\mathrm{i}$ & 0.8 \\
Ring Width & $\mathrm{w}$ & 0.3 \\
Gap width & $\mathrm{g}$ & 1 \\
Thickness & $\mathrm{t}$ & \\
\hline
\end{tabular}

This structure is known to exhibit a TM-like mode with strong longitudinal electric field in the central region of the waveguide, at $5.86 \mathrm{GHz}$, this mode takes the form shown in Fig. 2, with a strong area of field where the beam propagates. At this frequency the structure has strong beam coupling parameters, a shunt impedance of $181 \mathrm{k} \Omega$, and a $\mathrm{R} / \mathrm{Q}$ of $36 \Omega$, which indicate that any electron beam propagating through the structure is likely to excite this mode. These simulations were performed using the eigen mode solver of CST Microwave studio, ${ }^{10}$ with a tetrahedral mesh of 10 cells per wavelength which provides accuracy to $<0.01 \%$.

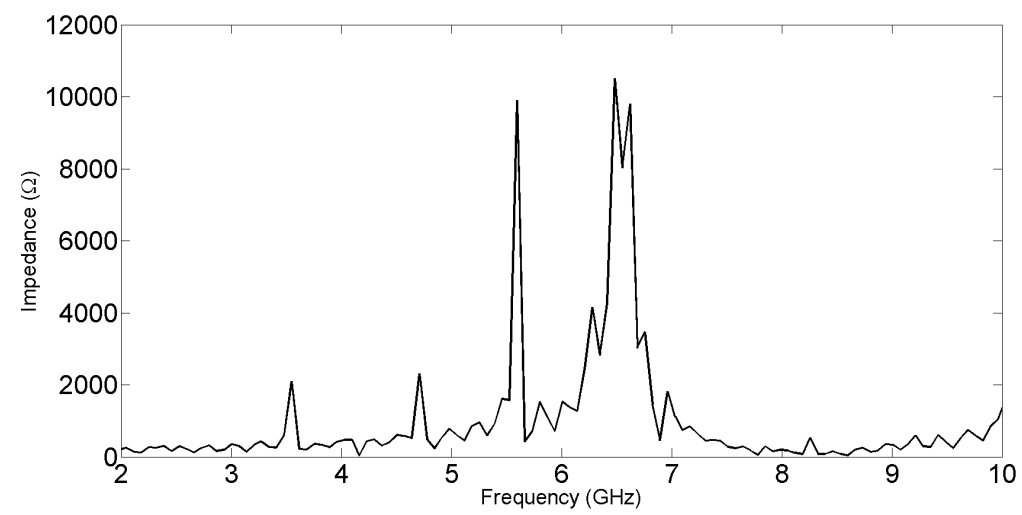

Figure 3. The longitudinal wake impedance of excited within the loaded waveguide structure, showing two strong excitation peaks at $5.6 \mathrm{GHz}$ and $6.49 \mathrm{GHz}$.

Prior to full PIC simulations, wakefield simulations have been performed using a hexahedral mesh of 15 cells per wavelength to ensure accuracy like the tetrahedral mesh. These simulations are performed to confirm the strong beam coupling, in these simulations a Gaussian electron beam was used, with a standard deviation bunch length of $\sigma=1.5 \mathrm{~mm}$, a charge of $1 \mathrm{nC}$ and an offset of $0.5 \mathrm{~mm}$ in $\mathrm{x}$ and y for the transverse wakes is used. Two strong excitations of the longitudinal wake impedance are observed in Figure. 3 for the structure when this beam propagates. The first at $5.60 \mathrm{GHz}$ exhibits an impedance of $9.6 \mathrm{k} \Omega$ and the second at $6.49 \mathrm{GHz}$ exhibits a strength of $10.5 \mathrm{k} \Omega$, the latter of these two modes corresponds to the TM-like mode found in electromagnetic simulations and agrees with the strong beam coupling parameters found analytically. In comparison to the longitudinal wakefield, the transverse wakefield has weak excitations, these occur at the same frequency as the longitudinal excitations however strength of these is much smaller and therefore no disruption of the beam is expected. 


\section{PARTICLE IN CELL SIMULATIONS}

Through the particle in cell (PIC) simulations we aim to obtain a comprehensive view of how the loaded waveguide structure and an electron beam interact, in particular, what fields the beam generates within the structure and whether these will correspond to the frequency at which the structure is a left handed medium. For these simulations, a section of the 4 layer loaded waveguide 40 resonators $(320 \mathrm{~mm})$ long will be used, this has been found long enough to observe relevant excitation strength without excessive increase of the computational burden. All simulations are performed using a hexahedral mesh of 15 cells per wavelength in CST particle studio, ${ }^{10}$ which has shown convergence of the results and thus ensures numerical accuracy. The simulation time for each set up is taken to be $50 \mathrm{~ns}$ and there exists an external analytical magnetic field of $0.5 \mathrm{~T}$ in the direction of the beam.

A single bunch is simulated for both high and low energy beams, with large $(2 \mathrm{~mm})$ and small $(250 \mu \mathrm{m})$ spot sizes, to determine and compare the proposed structure performance for different beams characteristics. The excitation in the longitudinal electric field correspond to modes with which the beam is interacting. These mode should be TM-like with strong longitudinal field in the region of the beam, and profiles similar to those found in the electromagnetic simulations and shown in Figure. 2. These simulations will highlight any modifications needed for operation in conjunction with low energy and high energy beams and will inform the beam tests we plan to perform on this structure at the Daresbury Laboratories.

\subsection{VELA Beam}

The versatile electron linear accelerator (VELA) ${ }^{11}$ is based on site at the Daresbury laboratory and is run by AsTeC and the STFC. VELA is a high performance facility able to deliver a highly stable, short pulse electron beam to a series of test enclosures, where the CSRR loaded waveguide will be located. For high energy beams, attention needs to be paid to the manufacture of the structure to prevent excessive out gassing that could damage the set up. VELA offers beams with energies of 4.5-5 MeV, with bunch length from 80 ps down to 3 ps, with minimal energy spread of $0.1-5 \%$. The specifics of the single bunch simulated are given in Table. 2 .

Table 2. The VELA beam parameters used to define the single Gaussian bunch in the PIC simulations.

\begin{tabular}{lr}
\hline Beam Parameter & Value and unit \\
\hline \hline Beam radius $\left(\sigma_{x y}\right)$ & $1.5 \mathrm{~mm}$ \\
Beam energy & $4.5 \mathrm{MeV}$ \\
Energy spread & $2 \%$ \\
Charge $(\mathrm{Q})$ & $10 \mathrm{nC}$ \\
Sigma $\left(\sigma_{z}\right)$ & $2.5 \mathrm{ps}$ \\
Cut off & $5 \mathrm{ps}$ \\
\hline
\end{tabular}

In all simulation performed, an electric field probe is placed in the center of the waveguide at a distance of 160 $\mathrm{mm}$ from the particle source along the z-direction, this probe monitors the longitudinal E-field component $E_{z}$. Excitation of the longitudinal E-field indicates interaction between the beam and the structure at that frequency, the field profile should be that of the TM-like mode found in electromagnetic simulations of the structure at 5.86 GHz.

Figure. 4 shows two peaks in the longitudinal field the strongest at $6.415 \mathrm{GHz}$ with a strength of $17.42 \mu \mathrm{V} / \mathrm{m}$ and a second lower energy peak of $11.8 \mu \mathrm{V} / \mathrm{m}$ at $6.34 \mathrm{GHz}$. Figure. 5 shows the profile of the z-component of the electric field that these peaks correspond to. It can be seen that the first peak at the lower frequency of $6.34 \mathrm{GHz}$ shown on the left, corresponds to the fundamental TM-like mode identified in the electromagnetic simulations. The second stronger peak at $6.415 \mathrm{GHz}$ shown on the right, corresponds to a higher order TM-like mode which is excited due to the small transversal size of the electron bunch, the higher order nature of this mode will increase the complexity of coupling. 


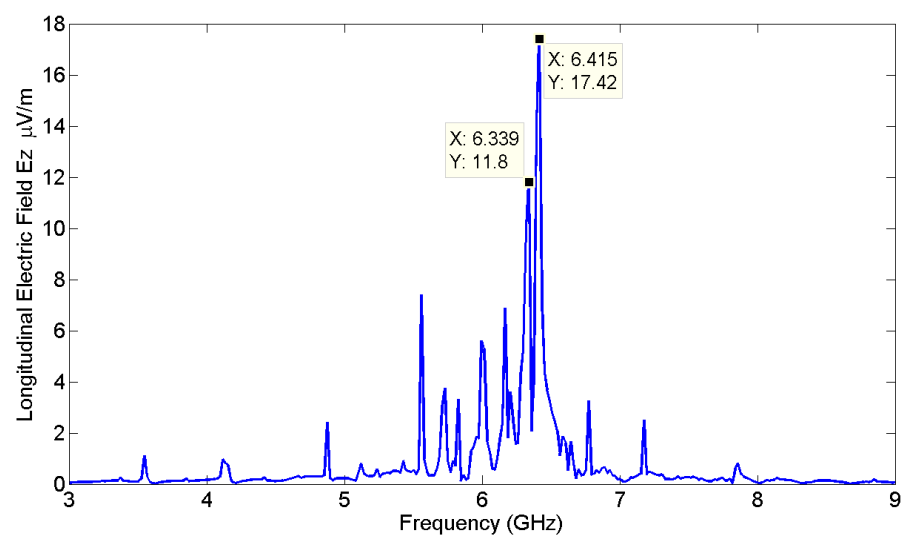

Figure 4. The longitudinal electric field at the probe placed in the centre of the waveguide when excited by the VELA beam.
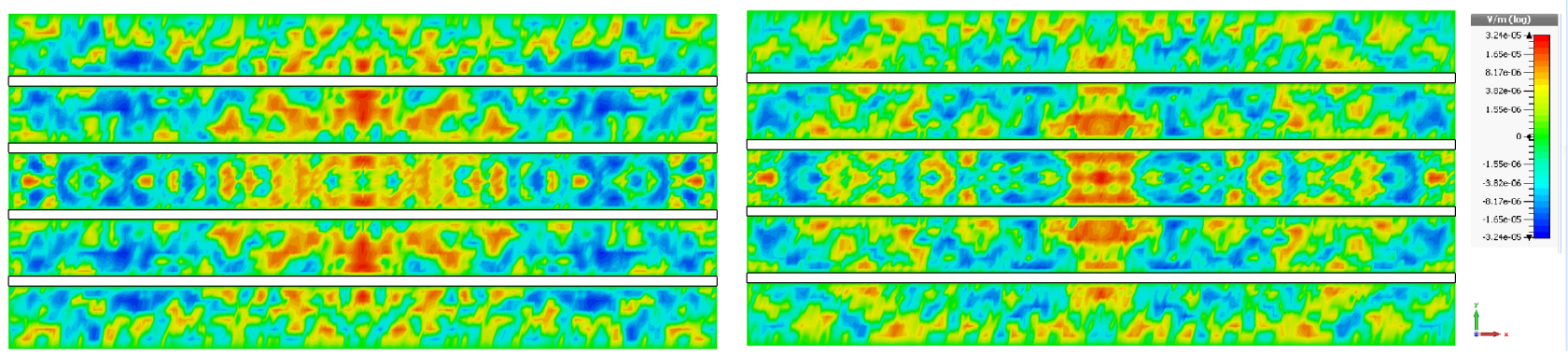

Figure 5. The fundamental (on the left) and the high-order (on the right) TM-like modes corresponding to the two excitation peaks found when the structure is excited by the VELA beam.

\subsection{AWAKE Beam}

Table 3. The AWAKE beam parameters used to define the single Gaussian bunch in the PIC simulations.

\begin{tabular}{lr}
\hline Beam Parameter & Value and unit \\
\hline \hline Beam radius $\left(\sigma_{x y}\right)$ & $250 \mu \mathrm{m}$ \\
Beam energy & $16 \mathrm{MeV}$ \\
Energy spread & $0.2 \%$ \\
Charge $(\mathrm{Q})$ & $10 \mathrm{nC}$ \\
Sigma $\left(\sigma_{z}\right)$ & $1 \mathrm{ps}$ \\
Cut off & $2 \mathrm{ps}$ \\
\hline
\end{tabular}

AWAKE is an advanced wakefield experiment proposed for $\mathrm{CERN}^{12}$ aiming to use a proton beam to drive a strong wakefield in plasma to accelerate a witness bunch of electrons. Here we focus on the beam used to generate the witness bunch, ${ }^{13}$ which is high intensity with short bunch lengths, hence has a very small beam radius. The full specification of the beam are given in Table. 3.

Figure. 6 shows the results of the $E_{z}$ field probe, it shows two clear peaks are found around the expected frequency, where synchronism with the beam is realized. The lower frequency peak at $6.312 \mathrm{GHz}$ has a strength of $18.76 \mu \mathrm{V} / \mathrm{m}$ and the higher frequency peak at $6.432 \mathrm{GHz}$ a strength of $22.19 \mu \mathrm{V} / \mathrm{m}$, these are stronger responses than those generated by the VELA bunch. Despite the much smaller beam radius, the enhanced interaction is due to the increase in beam energy and the shorter bunch length which leads to a more intense beam. 


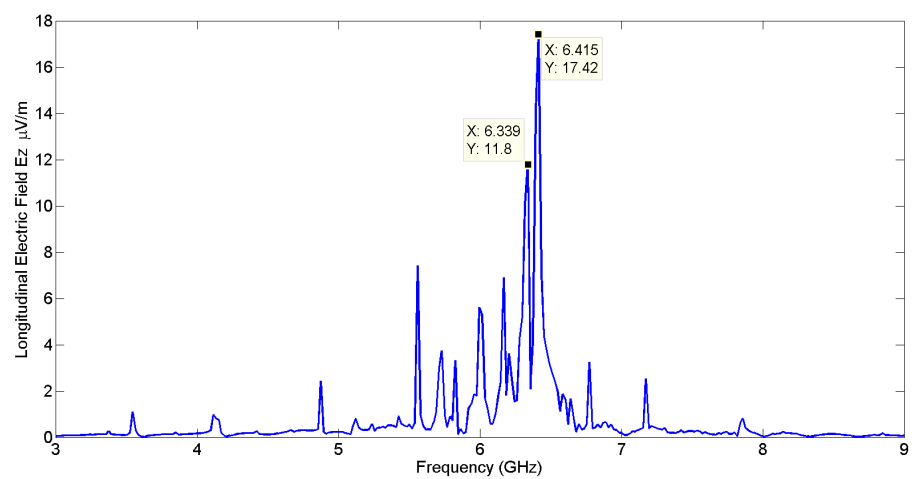

Figure 6. The longitudinal electric field at the probe placed in the centre of the waveguide for the AWAKE beam with $250 \mu \mathrm{m}$ spot size.
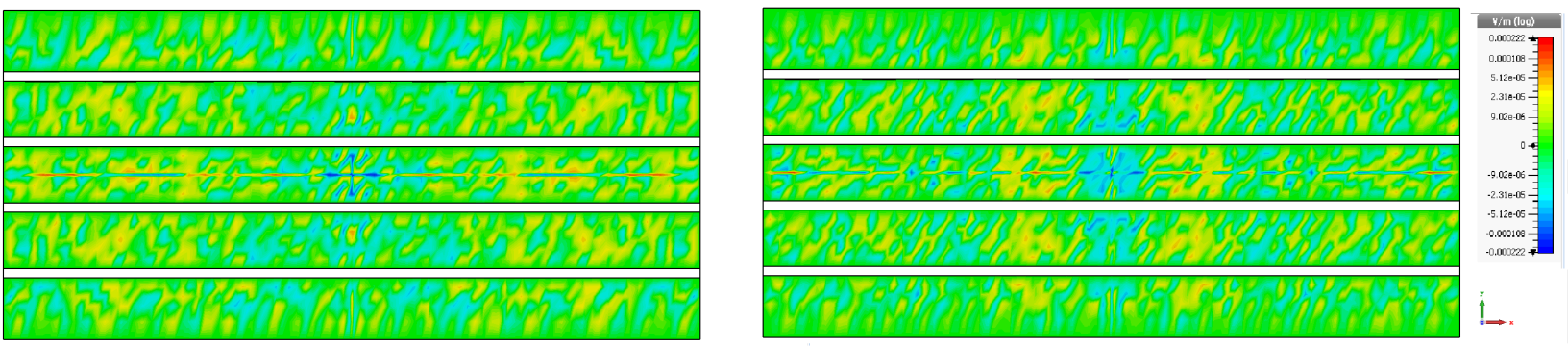

Figure 7. The fundamental (on the left) and the high-order (on the right) TM-like modes corresponding to the two excitation peaks found when the structure is excited by the AWAKE beam with $250 \mu \mathrm{m}$ spot size.

The field profiles of the two identified excitations are shown in Figure. 7, the field profiles are less defined than those found for the VELA beam. The lower frequency mode at $6.312 \mathrm{GHz}$ on the left, exhibits a weak version of the TM-like mode found in electromagnetic simulations, the central region of longitudinal field is apparent but less well defined, this is due to the small spot size of the beam. The higher frequency stronger mode at 6.432 $\mathrm{GHz}$ shown on the right, exhibits a higher order TM-like mode with strong regions close to the beam. These excitations show an interaction comparable to that of VELA, considering the increase in energy and intensity the response was expected to be higher.

\subsection{Kimball Physics Low Energy Commercial Beam}

Table 4. The beam parameters used to define the Gaussian beam in the PIC simulations for the low energy commercial Kimball gun.

\begin{tabular}{lr}
\hline Beam Parameter & Value and unit \\
\hline \hline Beam radius $\left(\sigma_{x y}\right)$ & $2 \mathrm{~mm}$ \\
Beam energy & $100 \mathrm{keV}$ \\
Energy spread & $0.4 \%$ \\
Charge $(\mathrm{Q})$ & $10 \mathrm{nC}$ \\
Sigma $\left(\sigma_{z}\right)$ & $1 \mathrm{~mm}$ \\
Cut off & $2 \mathrm{~mm}$ \\
\hline
\end{tabular}


The Kimball Physics beam is a low energy commercial beam, Kimball produce electron guns for energies from $5 \mathrm{keV}$ to $100 \mathrm{keV}$, with a range of specifications. ${ }^{14}$ For these PIC simulations their highest energy beam of $100 \mathrm{keV}$ is used. The bunch lengths here are given in terms of mm not ns, this is how the beam parameters are presented in the literature. The full specification of the beam are given in Table. 4.

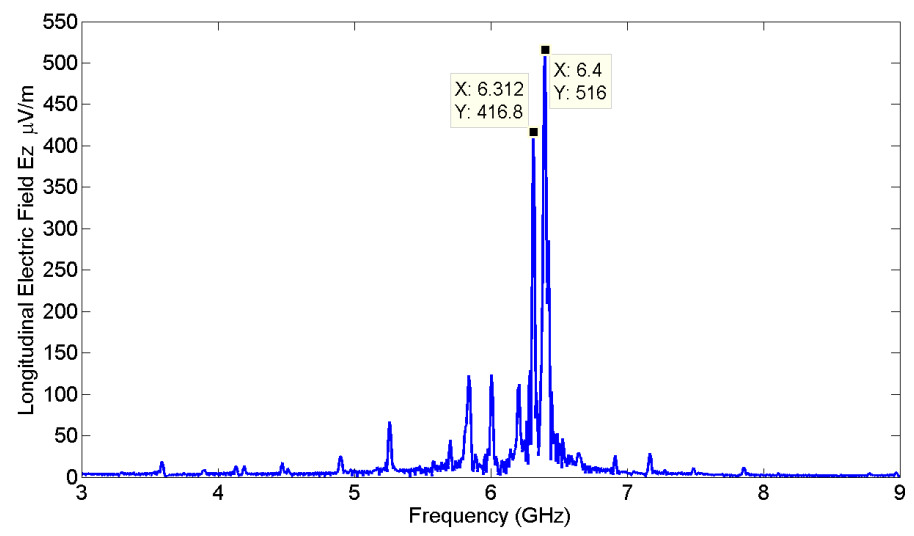

Figure 8. The longitudinal electric field at the probe placed in the centre of the waveguide for the Kimball beam.

Figure. 8 shows PIC simulations results in frequency domain with two distinct peaks of the $E_{z}$ field the first at $6.24 \mathrm{GHz}$ has a strength of $687 \mu \mathrm{V} / \mathrm{m}$ and the second lower peaks at $6.336 \mathrm{GHz}$ has a strength of $465 \mu \mathrm{V} / \mathrm{m}$. Neither of these peaks correspond with peaks generated by the other beams, they are lower in frequency and are of the highest magnitude seen in any set up, this lower frequency agrees with the idea that a lower energy beam gives rise to a low frequency response.

Figure. 9 shows the field profiles corresponding to these two spectral peaks confirming excitation of the fundamental and high-order TM-like modes. The lower frequency mode at $6.24 \mathrm{GHz}$ corresponds to the fundamental TM-like mode, much simpler to couple to than the fundamental TM-like modes found in other set ups. The higher frequency mode at $6.336 \mathrm{GHz}$ corresponds to a higher order TM-like mode that is weaker than the lower frequency peak.
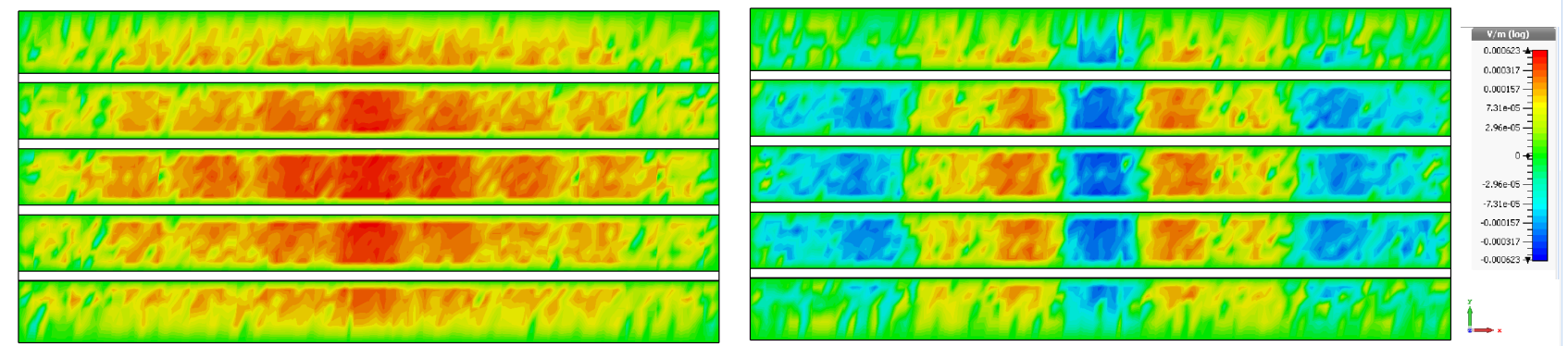

Figure 9. The fundamental (on the left) and the high-order (on the right) TM-like modes corresponding to the two excitation peaks found when the structure is excited by the kimball beam.

\section{CONCLUSIONS}

Three different electron beams have been considered for propagation through the structure and the performance of each beam has differed greatly. The Kimball low energy beam exhibited the strongest excitations of all the beams making it a desirable choice. The lower frequency mode that occurred with this beam exhibited an idea mode profile with a clear first order TM-like mode with strong central longitudinal field that would be easy to couple to, therefore a beam like this is ideal for preliminary low energy beam tests. Dispersion analysis of 
the structure will be performed to identify the nature of the mode when intersected by this non-relativistic beam.

The AWAKE beam and the VELA beams were of similar energy within the MeV range and exhibited excitations of similar magnitudes, weaker than those of Kimball beam, but not so weak that they cannot be considered. The results of the AWAKE beam are the least promising as the beam has a small spot size and thus should be much higher intensity than the VELA beam, however there is no significant improvement in results and the field profiles are less defined. This beam is unsuitable for the applications this structure is designed for due to the small spot size of the beam which results in a hybrid field profile. The VELA beam that we intend to perform higher energy tests with shows good response and the lower frequency mode corresponds to a mode that can be coupled to.

\section{ACKNOWLEDGMENTS}

Work supported by the STFC core grant ST/K520133/1 and the Institution of Engineering and Technology, whom we thank.

\section{REFERENCES}

[1] Pendry, J., Holden, A. J., Robbins, D. J., and Stewart, W. J., "Magnetism from conductors and enhanced nonlinear phenomena," Microwave Theory and Techniques, IEEE Transactions on 47(11), 2075-2084 (1999).

[2] Sharples, E. and Letizia, R., "Investigation of csrr loaded waveguide for accelerator applications," Journal of Instrumentation 9(11), P11017 (2014).

[3] Cherenkova, E. P., "The discovery of the cherenkov radiation," Nuclear Instruments and Methods in Physics Research Section A: Accelerators, Spectrometers, Detectors and Associated Equipment 595(1), 8-11 (2008). 2007 Proceedings of the Sixth International Workshop on Ring Imaging Cherenkov Detectors.

[4] Abo-Bakr, M., Feikes, J., Holldack, K., Wüstefeld, G., and Hübers, H.-W., "Steady-state far-infrared coherent synchrotron radiation detected at bessy ii," Phys. Rev. Lett. 88, 254801 (Jun 2002).

[5] Carr, G. L., Martin, M. C., McKinney, W. R., Jordan, K., Neil, G. R., and Williams, G. P., "High-power terahertz radiation from relativistic electrons," Nature 420(6912), 153-156 (2002).

[6] Gao, J. and Shen, F., "Radiation properties of cavity cherenkov radiation," Phys. Rev. A 73, 043801 (Apr 2006).

[7] Thompson, M. C., Badakov, H., Cook, A. M., Rosenzweig, J. B., Tikhoplav, R., Travish, G., Blumenfeld, I., Hogan, M. J., Ischebeck, R., Kirby, N., Siemann, R., Walz, D., Muggli, P., Scott, A., and Yoder, R. B., "Breakdown limits on gigavolt-per-meter electron-beam-driven wakefields in dielectric structures," Phys. Rev. Lett. 100, 214801 (May 2008).

[8] Sharples, E. and Letizia, R., "Development of metamaterials for cherenkov radiation based particle detectors," in [Proceedings international Particle Accelerator Conference IPAC 2014], TUPME038 (2014).

[9] Sharples, E. and Letizia, R., "Optimization of complementary split ring resonators for wakefield generation via metamaterial loaded waveguides," Physical Review special topics acclerators and beams (under review).

[10] [CST Microwave studio, 2014], CST Computer Simulation Technology AG, Germany (2014).

[11] STFC, "Astec - the versatile electron linear accelerator (vela)," (Acessed:2015-06-17).

[12] AWAKE, C., "Awake, advanced wakefield experiment," (Acessed:2015-06-23).

[13] Gschwendtner, E., Bracco, C., Meddahi, M., Petrenko, A., Doebert, S., Vincke, H., Osborne, J., Feldbaumer, E., Fedosseev, V., Hessler, C., et al., "The awake experimental facility at cern," tech. rep. (2014).

[14] KPI, K. P., "Kimball physics, electron gun systems comparison charts," (Acessed:2015-06-17). 